\title{
Nutrição enteral precoce e desfechos clínicos em pacientes de terapia intensiva
}

\author{
Early enteral nutrition and clinical outcomes in intensive care patients
}

\section{DOI: $10.37111 /$ braspenj.2020354009}

Janaína da Conceição Fernandes Gama'

Renata Quele Viana Silva ${ }^{1,2}$

Anne Caroline Brito Barroso 1,3

Luiz Gustavo Vieira Cardoso ${ }^{4}$

Matheus Lopes Cortes ${ }^{5}$

Vivian Francielle França ${ }^{4}$

\section{Unitermos:}

Nutrição enteral. Hospitalização. Respiração artificial.

\section{Keywords:}

Enteral nutrition. Hospitalization. Respiration, Artificial.

\section{Endereço para correspondência:}

Vivian Francielle França

Avenida Braulino Santos, 1601 - Edifício Noé de Oliveira Neto, 204 - Candeias - Vitória da Conquista, BA, Brasil - CEP: 45000-000

E-mail:vivianffranca@gmail.com

Submissão:

29 de abril de 2020

\section{Aceito para publicação}

10 de dezembro de 2020

\begin{abstract}
RESUMO
Introdução: O suporte nutricional precoce é estratégia terapêutica em pacientes críticos, contudo, tem se mostrado controverso em relação aos desfechos clínicos. O objetivo do estudo foi investigar os efeitos da terapia nutricional enteral (TNE) precoce e da oferta calórico-proteica inicial nos desfechos clínicos de pacientes em Unidades de Terapia Intensiva (UTI) de um hospital do Sudoeste Baiano. Método: Estudo de coorte prospectiva, aprovado pelo comitê de ética. Foram coletadas informações sobre triagem nutricional, avaliação antropométrica, necessidades nutricionais estimadas e metas nutricionais. $O$ tempo de introdução da TNE foi classificado como precoce, quando iniciada nas primeiras 48 horas da admissão e tardia. Foram monitorados o volume e as características da dieta enteral diariamente, bem como os tempos de internação na UTI e de ventilação mecânica e a mortalidade. Os pacientes foram acompanhados até a alta da UTI ou óbito. Para testar associação entre os desfechos tempo de ventilação mecânica e permanência na UTI e as variáveis nutricionais foi empregada a regressão linear, enquanto para mortalidade, a regressão logística. Resultados: Foram incluídos 88 pacientes, dos quais $96,6 \%$ apresentaram risco nutricional na admissão, determinado pela gravidade do quadro. A TNE precoce foi recebida por 67 pacientes, com média de $39 \pm 11,69$ horas para o início e variação significativa em relação à TNE tardia $(77,76 \pm 32,11$ horas), sem associação com os desfechos. As médias calóricas e proteicas recebidas nos três primeiros dias da TNE foram significativamente maiores no grupo TNE precoce $(p=0,000)$, as quais se associaram com maior tempo de permanência em UTI e de ventilação mecânica, mesmo com frequência elevada de inadequação proteica. Nenhum paciente da TNE tardia apresentou adequação proteica até o terceiro dia. Conclusão: A TNE precoce não foi associada aos desfechos clínicos de pacientes críticos, contudo a maior oferta de energia elevou os tempos de permanência em UTI e de ventilação mecânica.
\end{abstract}

\section{ABSTRACT}

Introduction: Early nutritional support is a therapeutic strategy in critically ill patients, however it has been shown to be controversial in relation to clinical outcomes. The aim of the study was to investigate the effects of early enteral nutritional therapy (ENT) and the initial caloric-protein supply on the clinical outcomes of patients in Intensive Care Units (ICU) of a hospital in Southwest Bahia. Methods: Prospective cohort study approved by the ethics committee. Information was collected on nutritional screening, anthropometric assessment, estimated nutritional needs and nutritional goals. The time of introduction of NET was classified as early, when started in the first 48 hours of admission and late. The volume and characteristics of the enteral diet were monitored daily, as well as the length of stay in the ICU and mechanical ventilation and mortality. Patients were followed up until discharge from the ICU or death. To test the association between the outcomes of mechanical ventilation time and ICU stay and nutritional variables, linear regression was used, while, for mortality, logistic regression. Results: 88 patients were included, of which $96.6 \%$ had nutritional risk at admission, determined by the severity of the condition. Early NET was received by 67 patients, with a mean of $39 \pm 11.69$ hours to onset and significant variation in relation to late NET (77.76 \pm 32.11 hours), with no association with outcomes. The caloric and protein averages received in the first three days of NET were significantly higher in the early NET group ( $p$ $=0.000$ ), which were associated with longer ICU stays and mechanical ventilation, even with a high frequency of protein inadequacy. No late ENT patient presented protein adequacy until the third day. Conclusion: Early NET was not associated with the clinical outcomes of critically ill patients, however, the greater energy supply increased the ICU stay and mechanical ventilation times.

1. Especialista em Residência Multiprofissional em Urgência, Hospital Geral de Vitória da Conquista, Universidade Federal da Bahia (UFBA), Vitória da Conquista, BA, Brasil.

2. Nutricionista do Hospital Geral de Vitória da Conquista (HGVC), Vitória da Conquista, BA, Brasil.

3. Nutricionista da NUTRA - representantes comerciais e agentes do comércio de mercadorias em geral não especializado, Vitória da Conquista, BA, Brasil.

4. Doutor (a) e docente do curso de Nutrição e do Programa de Residência Multiprofissional em Urgência da Universidade Federal da Bahia (UFBA), Vitória da Conquista, BA, Brasil.

5. Mestre e docente do curso de Nutrição e do Programa de Residência Multiprofissional em Urgência da Universidade Federal da Bahia (UFBA), Vitória da Conquista, BA, Brasil. 


\section{INTRODUÇÃO}

Nas últimas décadas, o suporte nutricional tem emergido como elemento fundamental no cuidado de pacientes críticos. As atuais diretrizes ${ }^{1-4}$ preconizam a nutrição enteral precoce e individualizada, iniciada dentro de 24 a 48 horas, na impossibilidade de manter ingestão alimentar voluntária. Embora existam fórmulas especializadas que promovam atenuação das complicações em pacientes críticos, o momento ideal para o início da terapia nutricional enteral (TNE) e as necessidades calóricas e proteicas destes pacientes são controversos ${ }^{5,6}$.

O suporte nutricional enteral precoce é visto como estratégia terapêutica proativa, que pode reduzir a gravidade da doença e complicações, diminuir o tempo de permanência hospitalar e da ventilação mecânica e tem impacto favorável no prognóstico dos pacientes ${ }^{7}$. Contudo, em pacientes hemodinamicamente instáveis, a TNE tem sido considerada problemática, pelo risco de isquemia intestinal e desvio do fluxo sanguíneo para o trato gastrointestinal, sem o aumento no débito cardíaco. Apesar dos impactos desses fenômenos na evolução clínica dos pacientes não estarem claros, a instabilidade hemodinâmica tem sido uma contraindicação ao início da TNE8 .

Claramente, a primeira semana após a admissão na UTI é o período mais controverso da hospitalização em relação à TNE. É sugerido evitar ou reduzir a alimentação nesse período, devido ao auge da gravidade da doença, porém, outro argumento válido se opõe a essa sugestão e recomenda que a alimentação seja fornecida na primeira semana, para promover os benefícios da TNE precoce?.

A despeito de alguns estudos que avaliaram a TNE precoce em terapia intensiva, permanecem ainda controversas algumas definições, razão que motivou a realização desse trabalho, cujo objetivo foi investigar os efeitos da TNE precoce e da oferta calórico-proteica inicial nos desfechos clínicos de pacientes hospitalizados em unidades de terapia intensiva (UTI). Além disso, a maioria dos estudos encontrados na literatura sobre essa temática não traz correlação dessas informações com as adequações energéticas e proteicas da terapia nutricional instituída, conferindo originalidade ao trabalho.

\section{MÉTODO}

Trata-se de uma coorte prospectiva realizada nas UTI de um hospital do Sudoeste Baiano. Todos os pacientes internados nas UTI nesse período foram julgados quanto aos critérios de inclusão, pacientes com idade superior a 14 anos, com permanência mínima na UTI por dois dias e que tiveram o Termo de Consentimento Livre e Esclarecido (TCLE) assinado pelo responsável foram incluídos. Para pacientes menores de idade, acrescentou-se o termo de assentimento. Pacientes que receberam algum tipo de nutrição em ambiente hospitalar prévio à admissão na UTI, aqueles cuja nutrição inicial na UTI foi por via oral ou parenteral ou com prontuários incompletos, sem o SAPS 3 foram excluídos.
A previsão da amostra foi baseada em dados anteriores que mostravam que a média mensal de admissão era de 20 pacientes por unidade, totalizando 240 em seis meses de estudo. Foi considerada perda de 50\% das informações dos prontuários frente à constatação da falta de rotina dos profissionais em realizar o sistema prognóstico SAPS3 na admissão do paciente, variável fundamental para análise dos resultados. Desta forma, eram esperados 120 pacientes elegíveis.

Foi realizada triagem nutricional nas primeiras 24 horas admissionais pelo Nutritional Risk Screening (NRS) 2002. Este método é recomendado pela European Society for Parenteral and Enteral Nutrition (ESPEN) e pela American Society for Parenteral and Enteral Nutrition (ASPEN), para identificar o risco nutricional do paciente, de modo a verificar aqueles com maior probabilidade de se beneficiarem da terapia nutricional precoce. $\bigcirc$ paciente com pontuação igual ou superior a três foi considerado em risco nutricional ${ }^{2,3}$.

A avaliação antropométrica inicial foi realizada considerando o peso em quilogramas $(\mathrm{kg})$ estimado pela equação de Chumlea et al. ${ }^{10}$ ou o peso referido para os pacientes com idade inferior a 19 anos, visto que as fórmulas para estimativa de peso não contemplam indivíduos dessa faixa etária. Para todos os participantes, a estatura $(\mathrm{cm})$ foi estimada pela equação de Chumlea et al. ${ }^{11}$. $\bigcirc$ índice de massa corporal (IMC; kg/m²) foi calculado com base nos dados de peso e estatura estimados ou referidos e classificado conforme idade do paciente.

Dados epidemiológicos e clínicos foram coletados do prontuário dos pacientes com auxílio de um instrumento padronizado, no qual foram inseridas informações sobre idade, sexo, diagnóstico de internação na UTI e Simplified Acute Physiology Score III (SAPS 3) ${ }^{12}$. De acordo com os diagnósticos admissionais mais frequentes nessas unidades, os pacientes foram categorizados em pós-operatório, doenças neurológicas, doenças respiratórias ou outras causas, quando não se inseriam nas anteriores.

O tempo decorrido até a introdução da TNE foi coletado das fichas de controle de cada paciente utilizadas nas UTI. As necessidades energéticas e proteicas foram estimadas individualmente. A meta calórica foi de $30 \mathrm{kcal} /$ $\mathrm{kg} /$ dia para pacientes desnutridos, $25 \mathrm{kcal} / \mathrm{kg} / \mathrm{dia}$ para eutróficos e $15 \mathrm{kcal} / \mathrm{kg} /$ dia para pacientes com sobrepeso ou obesidade. A meta da oferta de proteínas foi de 1,2 a $2 \mathrm{~g} / \mathrm{kg} /$ dia de acordo o IMC $\mathrm{MC}^{2,13}$. A partir dos dados coletados das fichas de controle sobre os volumes de dieta administrados, foi realizada a conversão em calorias e proteínas, de acordo com a composição de cada fórmula de nutrição enteral, para cálculo da adequação individual do valor energético total (VET) e da ingestão de proteína na primeira semana de internação, sendo considerado adequado quando recebido, no mínimo, $80 \%$ do valor prescrito $^{4}$. 
Os pacientes foram agrupados de acordo com o tempo de introdução da TNE, embasados em recomendações ${ }^{2}$ e à semelhança de outros estudos que avaliaram a relação entre tempo de iniciação da terapia nutricional e desfechos clínicos em pacientes críticos ${ }^{6}$. $O$ primeiro grupo, TNE precoce, foi constituído pelos pacientes cuja dieta foi iniciada nas primeiras 48 horas após admissão em UTI e os demais pacientes constituíram o segundo grupo, considerado TNE tardia. As médias calóricas e proteicas foram calculadas considerando os três primeiros dias de recebimento da TNE, visto que a dieta foi iniciada em momentos diferentes da internação para cada paciente.

Os dados referentes à nutrição enteral foram analisados até a alta da UTI ou óbito, sendo considerado o período máximo para análise até o sétimo dia para aqueles pacientes que permaneceram por mais de uma semana em terapia intensiva. Esse período foi definido tendo em vista que as diretrizes sugerem que os benefícios da TNE precoce são alcançados na primeira semana de internação ${ }^{1-4}$, possibilitando a associação entre o tempo de introdução da dieta e os desfechos avaliados.

Os desfechos tempo de internação em UTI, tempo de ventilação mecânica, alta ou mortalidade na UTI foram computados com base nos dados referenciados em prontuário até o último dia de internação.

Foram realizadas análises descritivas para as variáveis clínicas e epidemiológicas, para os desfechos clínicos avaliados e variáveis de controle. Para esta finalidade, foram aplicados os testes de Kolmogorov-Smirnov, T de Student, ANOVA e Qui-quadrado de Pearson, ou correspondentes não paramétricos.

Foram realizadas análises multivariadas de associação entre a introdução precoce da TNE e os desfechos clínicos avaliados, bem como associação entre o valor energético médio recebido nos três primeiros dias da TNE tardia ou precoce com os tempos de permanência na UTI e de ventilação mecânica. Para associação entre o desfecho mortalidade e a TNE precoce foi empregada a regressão logística binária, com os resultados apresentados em odds ratio (OR). Para os desfechos tempo de internação e de ventilação mecânica, foi utilizada a regressão linear simples e múltipla, com os resultados apresentados em beta $(\beta)$. Foram realizadas análises brutas e ajustadas para as variáveis de controle que entraram no modelo a partir do teste de Wald ( $p$-valor $<0,20)$. O intervalo de confiança (IC) utilizado nas análises foi de $95 \%$. Foi utilizado o software Statistical Package for the Social Sciences (SPSS) ${ }^{\circledR}$ versão 21.0 .

O projeto foi aprovado pelo Comitê de Ética em Pesquisa do Instituto Multidisciplinar em Saúde da Universidade Federal da Bahia, consoante à Declaração de Helsinki e com as normas estabelecidas pela resolução $n^{\circ} 466 / 2012$ do Conselho Nacional de Saúde. Número de parecer 2.712.842 e CAAE 80799217.2.0000.5556.

\section{RESULTADOS}

Por conta do baixo número de pacientes elegíveis no período previsto do estudo, as coletas foram ampliadas para nove meses, de março a novembro de 2018. Durante este período, foram admitidos 471 pacientes, porém apenas 88 atenderam aos critérios de elegibilidade e foram incluídos no estudo, com perda superior a $80 \%$ da amostra, em especial pela ausência do registro do SAPS3.

A amostra foi composta, majoritariamente, por indivíduos do sexo masculino, faixa etária entre 19 e 59 anos e com diagnóstico de doenças neurológicas. Não houve diferença significativa do escore prognóstico SAPS 3 entre os grupos TNE precoce e tardia, indicando semelhante perfil de gravidade entre os mesmos (Tabelas 1 e 2).

Cerca de $97 \%$ dos pacientes apresentaram risco nutricional à admissão, embora quase metade deles estivesse com diagnóstico nutricional de sobrepeso ou obesidade. A TNE precoce foi recebida por $76 \%$ dos pacientes, com média de $39 \pm 11,69$ horas para o seu início, o que variou, significativamente, em

Tabela 1 - Características epidemiológicas e clínicas dos pacientes, segundo o tempo de introdução da Terapia Nutricional Enteral (TNE).

\begin{tabular}{|c|c|c|c|c|c|}
\hline \multirow[t]{2}{*}{ Variáveis } & \multicolumn{2}{|c|}{$\begin{array}{c}\text { TNE } \\
\text { Precoce } \\
(67) \\
\end{array}$} & \multicolumn{2}{|c|}{$\begin{array}{c}\text { TNE } \\
\text { Tardia } \\
(21) \\
\end{array}$} & \multirow[t]{2}{*}{ p-valor } \\
\hline & $\mathbf{N}$ & $\%$ & $\mathrm{n}$ & $\%$ & \\
\hline \multicolumn{6}{|l|}{ Sexo } \\
\hline Masculino & 44 & 65,7 & 13 & 61,9 & 0,753 \\
\hline Feminino & 23 & 34,3 & 8 & 38,1 & \\
\hline \multicolumn{6}{|l|}{ Faixa etária (anos) } \\
\hline$\leq 18$ anos & 6 & 9 & 2 & 9,5 & \\
\hline 19 a 59 anos & 43 & 64,1 & 12 & 57,2 & $0,832^{* *}$ \\
\hline$\geq 60$ anos & 18 & 26,9 & 7 & 33,3 & \\
\hline \multicolumn{6}{|l|}{ Diagnóstico admissional } \\
\hline Pós-operatório & 8 & 11,9 & 3 & 14,3 & \\
\hline Doenças neurológicas & 39 & 58,3 & 10 & 47,6 & $0,844^{\star \star}$ \\
\hline Doenças respiratórias & 10 & 14,9 & 2 & 9,5 & \\
\hline Outras causas & 10 & 14,9 & 6 & 28,6 & \\
\hline
\end{tabular}

\begin{tabular}{lccccc}
\hline $\begin{array}{l}\text { Estado nutricional } \\
\text { admissional }\end{array}$ & & & & & \\
$\quad$ Eutrofia & 25 & 37,4 & 10 & 47,6 & \\
$\quad \begin{array}{l}\text { Desnutrição } \\
\text { Sobrepeso/ Obesidade }\end{array}$ & 8 & 11,9 & 2 & 9,5 & $0,725^{* *}$ \\
\hline Risco nutricional & 34 & 50,7 & 9 & 42,9 & \\
$\quad$ Sem risco nutricional & 2 & 3 & 1 & 4,8 & $0,695^{*}$ \\
$\quad$ Com risco nutricional & 65 & 97 & 20 & 95,2 & \\
\hline Adequação calórica da TNE & & & & & \\
$\quad \geq 80 \%$ & 15 & 22,4 & 1 & 4,8 & $0,068^{*}$ \\
$\quad<80 \%$ & 52 & 77,6 & 20 & 95,2 & \\
\hline Adequação proteica da TNE & & & & & \\
$\quad \geq 80 \%$ & 8 & 11,9 & & & $0,097^{*}$ \\
$\quad<80 \%$ & 59 & 88,1 & 21 & 100 & \\
\hline Mortalidade & & & & & \\
$\quad$ Alta & 49 & 73,1 & 15 & 71,4 & $0,878^{*}$ \\
$\quad$ Óbito & 18 & 26,9 & 6 & 28,6 & \\
\hline
\end{tabular}

Adequação calórica da TNE considera a $1^{a}$ semana de internação. *Qui-quadrado de Pearson. ${ }^{\star \star}$ Teste Exato de Fisher. Nível de significância de 5\%. 
Tabela 2 - Médias das principais variáveis do estudo.

\begin{tabular}{|c|c|c|c|c|c|}
\hline \multirow[t]{2}{*}{ Variáveis } & \multicolumn{2}{|c|}{$\begin{array}{c}\text { TNE } \\
\text { Precoce (67) }\end{array}$} & \multicolumn{2}{|c|}{$\begin{array}{c}\text { TNE } \\
\text { Tardia (21) } \\
\end{array}$} & \multirow[t]{2}{*}{$\mathrm{p}$-valor } \\
\hline & $\mathbf{N}$ & $\%$ & $\mathbf{n}$ & $\%$ & \\
\hline Horas para iniciar TNE & 39,1 & 11,6 & 77,7 & 32,1 & 0,00 \\
\hline Dias de internação na UTI & 15,0 & 9,9 & 12,1 & 5,3 & 0,08 \\
\hline Dias de ventilação mecânica & 11,8 & 9,6 & 7,8 & 4,9 & 0,01 \\
\hline SAPS* 3 & 58,8 & 13,8 & 54,8 & 12,5 & 0,23 \\
\hline $\begin{array}{l}\text { Calorias nos três primeiros } \\
\text { dias da TNE }\end{array}$ & 894,4 & 369,7 & 536,3 & 420 & 0,00 \\
\hline $\begin{array}{l}\text { Calorias na } 1^{\text {a }} \text { semana de } \\
\text { internação }\end{array}$ & 1118,4 & 437,4 & 698,6 & 420,4 & 0,00 \\
\hline $\begin{array}{l}\text { Proteínas nos três primeiros } \\
\text { dias da TNE }\end{array}$ & 43,7 & 19,4 & 25,5 & 20,1 & 0,00 \\
\hline $\begin{array}{l}\text { Proteínas na } 1^{a} \text { semana de } \\
\text { internação }\end{array}$ & 54,7 & 22,3 & 33,4 & 20,1 & 0,00 \\
\hline
\end{tabular}

relação ao período de início da TNE tardia $(77,76 \pm 32,11$ horas) ( $p=0,000)$. As médias de calorias e proteínas recebidas nos três primeiros dias da TNE, assim como ao final da primeira semana de internação, foram significativamente maiores no grupo TNE precoce $(p=0,000)$, contudo, em ambos os grupos, a maioria dos pacientes não obteve adequação calórica e, principalmente, proteica, destacando-se que nenhum paciente do grupo TNE tardia alcançou adequação de proteínas na primeira semana (Tabelas 1 e 2).

Quando comparamos as necessidades energéticas e proteicas com o valor médio ofertado ao final da primeira semana de internação, estratificadas de acordo com o estado nutricional, observamos um elevado déficit em todos os grupos, sendo de 53,3\%, 48\% e 60,4\% de calorias, e de $52,2 \%$, 45\% e 44,7\% de proteínas, respectivamente, para pacientes desnutridos, eutróficos e com sobrepeso.

A prevalência de mortalidade foi ligeiramente maior no grupo TNE tardia, porém sem significância estatística. $\bigcirc$ grupo que recebeu TNE precoce teve maiores períodos de internação em UTI e de ventilação mecânica, com diferença significativa para este último $(p=0,01)$ (Tabelas 1 e 2). As análises multivariadas não evidenciaram associação entre introdução precoce da TNE e os desfechos clínicos avaliados, mesmo após ajuste para as variáveis de controle (Tabela 3). As análises de associação entre a média de calorias nos três primeiros dias de recebimento da TNE e ao final da primeira semana de internação, independentemente do tempo de introdução da dieta, explicaram o aumento do tempo de permanência na UTI e do tempo de ventilação mecânica, mesmo após o ajuste para as variáveis SAPS3 e risco nutricional. Ao extrapolar as análises para o desfecho mortalidade na UTI e as variáveis relacionadas ao recebimento energético não foi observada associação (Tabela 4).

Tabela 3 - Relação entre mortalidade, tempo de internação e tempo de ventilação mecânica com o tempo de introdução da terapia nutricional enteral (TNE) precoce.

\begin{tabular}{lcccccc}
\hline Variáveis & \multicolumn{3}{c}{ Análises brutas } & \multicolumn{2}{c}{ Análises ajustadas } \\
\cline { 2 - 7 } & OR/ & IC (95\%) & p-valor & OR/ & IC (95\%) & p-valor \\
\hline Mortalidade na UTI & $+1,089$ & 0,$366 ; 3,239$ & 0,87 & $+1,355$ & $0,429-4,278$ \\
Dias de internação na UTI & $-2,964$ & $-7,501 ; 1,572$ & 0,19 & $+0,981$ & $-4,761 ; 6,723$ & 0,60 \\
Dias de ventilação mecânica & $-4,041$ & $-8,406 ; 0,324$ & 0,06 & $-1,632$ & $-6,703 ; 3,439$ & 0,52 \\
\hline
\end{tabular}

Regressão logística binária (odds ratio - OR) para mortalidade na UTI; Regressão linear (Beta - $\beta$ ) para dias de internação na UTI e dias de ventilação mecânica; Análises ajustadas para SAPS 3. Para ajuste, $p$-valor $<0,20$ pelo Teste de Wald. Nível de significância adotado nas análises foi $5 \%$.

Tabela 4 - Relação entre tempo de internação, tempo de ventilação mecânica, mortalidade na UTI com o valor calórico dos três primeiros dias de recebimento da TNE e ao final da primeira semana de internação na UTI.

\begin{tabular}{|c|c|c|c|c|c|c|}
\hline \multirow{2}{*}{ Variáveis } & \multicolumn{3}{|c|}{ Análises brutas } & \multicolumn{3}{|c|}{ Análises ajustadas } \\
\hline & OR/ & IC (95\%) & p-valor & OR/ & IC (95\%) & p-valor \\
\hline \multicolumn{7}{|l|}{ Dias de internação na UTI } \\
\hline Calorias nos três primeiros dias da TNE & $+0,007$ & $+0,001 ;+0,012$ & 0,00 & $+0,007$ & $+0,002 ;+0,011$ & 0,00 \\
\hline Calorias na $1^{\text {a }}$ semana de internação & $+0,009$ & $+0,006 ;+0,013$ & 0,00 & $+0,009$ & $+0,005 ;+0,013$ & 0,00 \\
\hline \multicolumn{7}{|l|}{ Dias de ventilação mecânica } \\
\hline Calorias nos três primeiros dias da TNE & $+0,006$ & $+0,001 ;+0,010$ & 0,01 & $+0,006$ & $+0,001 ;+0,010$ & 0,01 \\
\hline Calorias na $1^{\text {a }}$ semana de internação & $+0,005$ & $+0,005 ;+0,012$ & 0,00 & $+0,008$ & $+0,005 ;+0,012$ & 0,00 \\
\hline \multicolumn{7}{|l|}{ Mortalidade na UTI } \\
\hline Calorias nos três primeiros dias da TNE & $+0,000$ & $+0,999 ;+1001$ & 0,59 & $+0,009$ & $+0,998 ;+1,001$ & 0,58 \\
\hline Calorias na $1^{a}$ semana de internação & $+0,000$ & $+0,999 ;+1,001$ & 0,41 & $-0,0001$ & $+0,998 ;+1,001$ & 0,33 \\
\hline
\end{tabular}

Regressão logística binária (odds ratio - OR) para mortalidade na UTI; Regressão linear bruta e múltipla: Beta ( $\beta$ ). Análises ajustadas para o SAPS 3 e o Risco Nutricional. Nível de significância das análises de $5 \%$. 


\section{DISCUSSÃO}

Neste estudo, não foi encontrada associação entre a TNE precoce e melhores desfechos clínicos, contrapondo-se a isso, foi mostrado que a maior oferta energética elevou os tempos de permanência em UTI e de ventilação mecânica. Verificouse que a maioria dos pacientes recebeu TNE precoce de acordo com as recomendações ${ }^{1-4}$ e semelhantemente a outros estudos que avaliaram a TNE precoce em pacientes de terapia intensiva ${ }^{6,14}$.

Diferentemente dos nossos resultados, no estudo de coorte envolvendo 1.174 pacientes de UTI mostrou que os pacientes com TNE precoce tiveram redução significativa da mortalidade, do tempo de permanência em UTI e do tempo de ventilação mecânica quando comparados aos que receberam TNE tardia ${ }^{15}$. Contudo, as análises de subgrupos indicaram que o efeito benéfico da nutrição precoce foi apenas para pacientes mais críticos, dificultando a comparação com nossos achados, cujo perfil de gravidade pelo SAPS 3 foi semelhante entre os grupos. Este achado é importante, pois levanta a necessidade de as UTI utilizarem outros indicadores para a gravidade além do SAPS 3.

Por outro lado, uma metanálise comparou TNE precoce com outros tipos de suporte nutricional em pacientes na UTI e não encontrou redução da mortalidade e do tempo de ventilação mecânica, mostrando, apenas, tendência na redução do tempo de permanência na UTI'16. De igual modo, a European Society of Intensive Care Medicine (ESICM) divulgou não ter encontrado evidências de que a TNE precoce reduza a mortalidade em pacientes com traumas neurológicos, abdominais ou submetidos a cirurgias do trato gastrointestinal, reforçando os achados do nosso estudo, considerando que tais diagnósticos prevaleceram na amostra avaliada.

Os pacientes mais graves que não receberam TNE precoce tiveram medidas de suporte mais intensivo, tais como drogas vasoativas e procedimentos diagnósticos. Logo, pode ter sido essa conduta que impactou positivamente no tempo de ventilação mecânica e de internação na UTI de pacientes do grupo TNE tardia e não a ausência do suporte nutricional precoce. Houve uma tentativa de controlar esse viés por meio das análises ajustadas para gravidade da doença e risco nutricional, mas ainda assim não foi observada associação.

Quanto ao risco nutricional, a maioria dos pacientes apresentou escore elevado na NRS (2002), mesmo frente a $47 \%$ dos pacientes terem sido admitidos com sobrepeso ou obesidade. É reconhecido que o estado inflamatório e hipercatabólico dos pacientes críticos somados à gravidade da doença aceleram o processo de desnutrição intra-hospitalar. Neste sentido, a recente diretriz brasileira de terapia nutricional sugere que estes sejam fatores importantes para definir quem se beneficiará da nutrição precoce ${ }^{4}$.
Outra constatação desse estudo foi a associação entre a maior oferta de calorias nos três primeiros dias de TNE e os maiores tempos de internação e de ventilação mecânica, mesmo após o ajuste para o risco nutricional. Os pacientes que receberam TNE precoce tiveram aporte calórico nos três primeiros dias de dieta significativamente maior do que os pacientes cuja TNE foi tardia. A progressão do volume da dieta no grupo TNE precoce não ocorreu de forma gradativa, conforme preconizado ${ }^{1-4}$, aumentando cerca de quatro vezes a cada 24 horas, o que pode ter sido deletério para os pacientes alimentados precocemente, mesmo quando o paciente se encontrava em risco nutricional. Em concordância com estes achados, outros autores apontam que a ingestão limitada de nutrientes pode ser benéfica durante as primeiras 72 horas da doença aguda ${ }^{7,17}$. Em outro estudo foi observado que os pacientes que atingiram a meta calórica prevista nas primeiras horas de internação na UTI não tiveram melhores desfechos ${ }^{18}$. Uma metanálise mostrou que a oferta inicial de calorias moderada por via enteral foi mais benéfica quando comparada ao aporte calórico mínimo ou máximo ${ }^{19}$. Argumentos contra a ingestão elevada de calorias durante a fase inicial da doença crítica pautamse na produção endógena de glicose que corresponde a $50 \%$ a $75 \%$ da necessidade energética e na supressão da autofagia por macronutrientes exógenos ${ }^{20}$, levando ao aumento da morbidade ${ }^{21}$.

As formulações de TNE recebidas pelos pacientes do estudo tinham 1,0 a 1,2 kcal/mL e 1,0 g de proteína/ $\mathrm{mL}$. É sabido que as formulações enterais com a característica "normocalórica" se adequam mais à fase aguda da doença por controlar melhor a oferta energética ao paciente, no entanto, são poucas as formulações enterais que apresentam em sua constituição uma oferta proteica satisfatória.

Esse fato foi claramente observado neste estudo, em que a maioria dos pacientes em TNE precoce $(88,1 \%)$ e todos os pacientes em TNE tardia apresentaram inadequação da ingestão proteica ao final da primeira semana de hospitalização na UTI. Além disso, de forma alarmante, nenhum dos grupos apresentou média de oferta de proteínas $/ \mathrm{kg} /$ dia ajustada às metas nos três primeiros dias de TNE. Em outro estudo 22 foi observado que 70,2\% dos pacientes críticos atingiram a meta proteica proposta $(1,2-2,0 \mathrm{~g} / \mathrm{kg} / \mathrm{dia})$ de acordo com a necessidade nutricional estimada em até 3 dias, mas apenas $46,8 \%$ deles consumiram pelo menos $1,2 \mathrm{~g} / \mathrm{kg} /$ dia de proteína durante o internamento na UTI. Os autores também verificaram associações relevantes do aporte proteico com a mortalidade dos pacientes críticos.

Acrescenta-se, ainda, a necessidade de um olhar mais apurado aos pacientes que são admitidos na UTI com 
diagnóstico de desnutrição, uma vez que se sabe que o estado nutricional deficiente é fator agravante ao prognóstico do paciente ${ }^{4}$. Entre os pacientes obesos, embora o déficit energético tenha sido maior, este foi o grupo que teve o menor déficit proteico.

Quanto ao método de infusão da dieta foi verificado que os pacientes foram submetidos à TNE contínua. A administração contínua configura-se como prática comum em hospitais de todo o mundo, porém, seu uso é controverso, sob o argumento de que a administração contínua contraria a fisiologia normal e limita a síntese proteica, por alterar a resposta hormonal à ingestão de nutrientes ${ }^{23}$.

Como limitação do estudo destacamos a utilização do SAPS 3 como único indicador de gravidade. Esse escore avalia o uso de substâncias vasoativas somente no momento anterior à UTI, podendo ter subestimado a instabilidade hemodinâmica em alguns pacientes que receberam a TNE precoce ${ }^{16}$. Além desse fator, as necessidades dos pacientes podem ter sido sub ou superestimadas, pela dificuldade de avaliar a necessidade energética no paciente crítico por equações preditivas e pela ausência de fidedignidade no uso do peso referido ou estimado por equações disponíveis ${ }^{12}$. A população do estudo foi muito abrangente quanto à idade, o que, apesar do perfil de gravidade ter sido semelhante, pode ter sido um viés para os resultados. Outras limitações foram o caráter observacional da pesquisa e o número de pacientes que compuseram a amostra, por optarmos em adotar rigor metodológico para minimizar os vieses, restringindo o tamanho da amostra.

Embora os resultados continuem controversos, o estudo contribui para solidificar a informação de que a terapia nutricional é fundamental para prevenir a desnutrição, reduzir complicações e a mortalidade hospitalar, contudo, deve ser cautelosa em não ofertar uma sobrecarga energética ao paciente crítico. Aliado a isso, o estudo traz à tona um problema comum, o da inadequação proteica, ao passo de que está bem estabelecida a necessidade de priorizar o uso de formulações enterais hiperproteicas em prol de reduzir riscos potenciais ao paciente crítico $^{24,25}$.

\section{CONCLUSÃO}

Não foi observada associação entre nutrição enteral precoce e melhores desfechos clínicos em pacientes de terapia intensiva, entretanto, a maior oferta de calorias na fase aguda da doença crítica associou-se com o maior tempo de permanência em UTI e de dependência da ventilação mecânica. Os resultados demonstraram que a inadequação proteica foi frequente nos pacientes críticos, mesmo para aqueles com TNE precoce. Esse achado é alarmante, pois é conhecido que a baixa oferta proteica é fator de risco aos desfechos negativos em pacientes graves.

\section{AGRADECIMENTOS}

Agradecemos ao Hospital Geral de Vitória da Conquista, por nos ter confiado o seu campo de pesquisa, e ao Programa de Residência Multiprofissional em Urgência da Universidade Federal da Bahia, pela dedicação e oportunidades. Agradecemos pela ajuda das nutricionistas Júnia Maria e Carine Luz na coleta dos dados.

\section{REFERÊNCIAS}

1. Dhaliwal R, Cahill N, Lemieux M, Heyland DK. The Canadian critical care nutrition guidelines in 2013: an update on current recommendations and implementation strategies. Nutr Clin Pract. 2014;29(1):29-43.

2. McClave SA, Taylor BE, Martindale RG, Warren MM, Johnson DR, Braunschweig C, et al; Society of Critical Care Medicine; American Society for Parenteral and Enteral Nutrition. Guidelines for the provision and assessment of nutrition support therapy in the adult critically ill patient: Society of Critical Care Medicine (SCCM) and American Society for Parenteral and Enteral Nutrition (A.S.P.E.N.). JPEN J Parenter Enteral Nutr. 2016;40(2):159-211.

3. Singer P, BlaserAR, Berger MM, Alhazzani W, Calder PC, Casaer MP, et al. ESPEN guideline on clinical nutrition in the intensive care unit. Clin Nutr. 2019;38(1):48-79.

4. Campos ACL, Matsuba CST, Van Aanholt DPJ, Nunes DSL, Toledo DO, Rocha EEM, et al. Diretrizes brasileiras de terapia nutricional. Braspen J. 2018;33(Supl 1).

5. Marik PE. Is early starvation beneficial for the critically ill patient? Curr Opin Clin Nutr Metab Care. 2016;19(2):155-60.

6. Padilla PF, Martínez G, Vernooij RWN, Urrútia G, Figuls MRI, Cosp XB. Early versus delayed enteral nutrition support for critically ill adults. Cochrane Libr. 2016:1-21.

7. Blaser AR, Starkopf J, Alhazzani W, Berger MM, Casaer MP, Deane AM, et al; ESICM Working Group on Gastrointestinal Function. Early enteral nutrition in critically ill patients: ESICM clinical practice guidelines. Intensive Care Med. 2017;43(3):380-98.

8. Reitam Blaser A, Starkopf J, Alhazzani W, Berger MM, Casaer MP, Deane AM, et al. Early enteral nutrition in critically ill patients: ESICM clinical practice guidelines. Intensive Care Med. 2017;43(3):380-98.

9. McClave SA, Codner P, Patel J, Hurt RT, Allen K, Martindale RG. Should we aim for full enteral feeding in the first week of critical illness? Nutr Clin Pract. 2016;31(4):425-31.

10. Chumlea WC, Guo S, Roche AF, Steinbaugh ML. Prediction of body weight for the nonambulatory elderly from anthropometry. J Am Diet Assoc. 1988;88(5):564-8.

11. Chumlea WC, Guo SS, Steinbaugh ML. Prediction of stature from knee height for black and white adults and children with application to mobility-impaired or handicapped persons. J Am Diet Assoc. 1994;94(12):1385-8.

12. Bueno HL, Biatto JF. Epidemiologia e validação de escore prognóstico em UTI mista do norte do Paraná. Rev Uningá Review. 2018;22(3):23-9.

13. Lipschitz DA. Screening for nutritional status in the elderly. Prim Care. 1994;21(1):55-67.

14. Machado RR, Caruso L, Lima PA, Damasceno NR, Soriano FG. Nutrition therapy in sepsis: characterization and implications for clinical prognosis. Nutr Hosp. 2015;32(3):1281-8.

15. Khalid I, Doshi P, DiGiovine B. Early enteral nutrition and outcomes of critically ill patients treated with vasopressors and mechanical ventilation. Am J Crit Care. 2010;19(3):261-8. 
16. Tian F, Heighes PT, Allingstrup MJ, Doig GS. Early enteral nutrition provided within 24 hours of ICU admission: a metaanalysis of randomized controlled trials. Crit Care Med. 2018;46(7):1049-56.

17. Arabi YM, Aldawood AS, Haddad SH, Al-Dorzi HM, Tamim HM, Jones G, et al. Permissive underfeeding or standard enteral feeding in critically ill adults. N Engl J Med. 2015;372(25):2398-408.

18. Allingstrup MJ, Kondrup J, Wiis J, Claudius C, Pedersen UG, Hein-Rasmussen R, et al. Early goal-directed nutrition versus standard of care in adult intensive care patients: the single-centre, randomised, outcome assessor-blinded EAT-ICU trial. Intensive Care Med. 2017;43(11):1637-47.

19. Choi EY, Park DA, Park J. Calorie intake of enteral nutrition and clinical outcomes in acutely critically ill patients: a metaanalysis of randomized controlled trials. JPEN J Parenter Enteral Nutr. 2015;39(3):291-300.
20. Dyck LV, Casaer MP, Gunst J. Autophagy and its implications against early full nutrition support in critical illness. Nutr Clin Pract. 2018;33(3):339-47.

21. Preiser JC, van Zanten AR, Berger MM, Biolo G, Casaer MP, Doig GS, et al. Metabolic and nutritional support of critically ill patients: consensus and controversies. Crit Care. 2015;19(1):35.

22. Santos HVD, Araújo IS. Impacto do aporte proteico e do estado nutricional no desfecho clínico de pacientes críticos. Rev Bras Ter Intensiva. 2019;31(2):210-6.

23. Patel JJ, Rosenthal MD, Heyland DK. Intermittent versus continuous feeding in critically ill adults. Curr Opin Clin Nutr Metab Care. 2018;21(2):116-20.

24. Badosa EL, Tahull MB, Casas NV, Sangrador GE, Méndez CF, Meseguer IH, et al. Hospital malnutrition screening at admission: malnutrition increases mortality and length of stay. Nutr Hosp. 2017;34(4):907-13.

25. HeylandDK, Weijs PJ, Coss-Bu JA, Taylor B, KristofAS, O'Keefe $\mathrm{GE}$, et al. Protein delivery in the intensive care unit: optimal or suboptimal? Nutr Clin Pract. 2017;32(1 suppl):585-71S.

Local de realização do estudo: Hospital Geral de Vitória da Conquista, Bahia durante a especialização das Residentes no Programa de Residência Multiprofissional em Urgência/Universidade Federal da Bahia - UFBA, Vitória da Conquista, BA, Brasil.

Conflito de interesse: Os autores declaram não haver. 